\title{
Cuarto Congreso Colombiano de Obstetricia y Ginecología
}

Entre los días 29 de noviembre y 3 de diciembre del pasado año de 1959, tuvo lugar en ja ciudad de Barranquilla, capital del Departamento del Atlántico, el Cuarto Congreso Colombiano de la especialidad, organizado por la Sociedad Colombiana de Obstetricia y Ginecología y la Sociedad de Obstetricia y Ginecología del Atlántico, seccional de la primera.

Por tratarse del más concurrido, y en el cual se trató un mayor número de temas (incluyendo los temas libres), cabe destacarlo como uno de los más importantes celebrados últimamente.

Los temas oficiales aprobados en el Congreso anterior celebrado en la ciudad de Ibagué en el año 1957, fueron: Hiperestrogenismo, Aborto, Leucorreas y Toxemia.

El relato del primer tema (hiperestrogenismo) fué presentado por la cátedra de Ginecología de la Universidad de Antioquia (Medellín) dirigida por el Prof. Pedro Nel Cardona; el correlato y comentario fue presentado por la cátedra de Ginecología de la Universidad de Caldas, y las correspondientes contribuciones al tema por los Dres. Fernando Cardona Arango, Jorge Amorocho y el Prof. Dr. Arturo Aparicio.

El relato sobre aborto fue presentado por los profesores Dres. Rafael Peralta G., Guillermo López Escobar, Miguel A. Fernández-Bastidas, Gonzalo Echeverry, Hernando Navas y Efraín González de las cátedras de Obstetricia de la Universirlad Nacional y Universidad Javeriana de Bogotá; como correlatores, comentaristas actuaron los Dres. Eduardo Acosta Bendeck y Hernando Echeverry (de Barranquilla). Contribuciones al tema presentadas por el Dr. Saulo Muñoz D. (U. del Valle), Fernando Cardona A. (U. de Antioquia), Gustavo Isaza Mejía (U. de Antioquia) Gerardo Chadid S. (U. del Valle).

El tema sobre Leucorreas fue presentado por los Dres. Isaías Arenas Buenahora, Manuel Rangel, y col de la ciudad de Bucaramanga y en nombre de la Sociedad de Obstetricia y Ginecología de Santander. Como comentaris. tas actuaron la cátedra de Ginecología de la Universidad de Antioquia del Prof. P. N. Cardona. Las contribuciones al tema estuvieron a cargo de los Dres. R. Vergara Támara (U. Javeriana-Bogotá), Eduardo Acosta Bendeck (Barranquilla), Gustavo Isaza Mejía (U. de Antioquia) y Rodulfo Camero y col. (Hospital San José - Bogotá).

La ponencia sobre toxemia fue presentada conjuntamente por la cátedra de Obstetricia y Ginecología de la Universidad del Valle dirigida por el Prof. J. Escobar Soto y la cátedra de Obstetricia de la Universidad Nacional del Prof. 
Dr. Carlos R. Silva Mojica en colaboración con los Dres. Jesús A. Gómez Palacino, Fernando Sánchez y demás personal docente. Las contribuciones al tema presentadas por los Dres. Miguel A. Fernández-Bastidas, Jorge Sarmiento y G. Bahamon (U. Javeriana), Saulo Muñoz D. (U. del Valle). Entre los temas libres cabe destacar los siguientes: "Estudio de diez años sobre endometriosis en Colombia" por el grupo del Prof. Hernando Amaya León en colaboración con todos los grupos hospitalarios de Bogotá. "Estudio sobre sarcomas uterinos" de los Dres. G. López-Escobar y McLean. "Técnica quirúrgica para tratar prolapso de cúpula vaginal post histerectomía” del Prof. Héctor Enrique Bernal. Los profesores extranjeros Martial Dumont (Lyon) y Piero Malcovati (Milán) presentaron sendos estudios sobre "abscobinemia y embarazo" y "método psicoprofiláctico para el parto”. La parte social se cumplió de acuerdo con el programa; iniciándose con la inauguración llevada a cabo en el lujoso Hotel del Prado, durante la cual el Presidente de la Sociedad Colombiana de Obstetricia y Ginecología, Prof. Dr. Héctor Enrique Bernal, pronunció un elocuente discurso.

\section{DISCURSO DEL PROF. DR. HECTOR ENRIQUE BERNAL}

\section{Presidente de la Sociedad Colombiana de Obstetricia y Ginecología}

\section{Distinguidos Colegas:}

"Nos congregamos hoy por cuarta vez para un acontecimiento trascendental en los fastos científicos de Colombia; al igual que en otras ramas de la medicina en la especialidad obstétrico-ginecológica se producen continuos adelantos y es necesario hacer un alto en el camino para realizar un balance de los progresos alcanzados: tal la razón de nuestra presencia en este magno certamen, al cual se siente integralmente vinculada la Sociedad Colombiana de Obstetricia y Ginecología.

En efecto, para la Sociedad que tengo el honor de presidir constituye ésta una de sus funciones esenciales: así la vimos, hace algo más de un lustro, convocando a la Primera Conver.ción de la especialidad en la ciudad capital; más tarde fué el fértil Valle el lugar de la cita, luego el sufrido Tolima y ahora el portal de la Patria.

Hemos venido a dialogar durante algunos días sobre la más noble de las especialidades médicas; aquella que se ocupa de los problemas y que trata de luchar contra las causas que ensombrecen la vida de la mujer; ciencia que, como lo dijera un ilustre maestro, "contribuye al estudio en su origen de nuestro propio ser" y "vela por la salud del sagrado recinto donde pasamos del no existir a la vida real, como quiera que contempla los órganos donde la madre naturaleza elabora la más perfecta de sus obras, el único ser del universo que se parece a Dios".

Hemos venido, Señoras que me escucháis, a rendir un tributo a la mujer personificada en vosotras; a aquella mujer en cuyo regazo, libre el alma de pesares y el corazón de inquietudes, dejábamos reposar nuestra cabeza: dichosos mil veces los que todavía podemos contemplarla con los ojos de la realidad! A nuestras madres, a nuestras esposas, a nuestras hijas, a nuestras hermanas rendimos este homenaje; a ellas que han sabido dulcificar las horas de 
nuestra vida. Desdichados los que denigran por sistema al sexo que llaman débil: en su corazón no se escribió jamás el más sublime de los vocablos.

Se nos ha convocado a una convención de especialidad, más, en tratándose de la nuestra, el concepto se ensancha y adquiere caracteres de universalidad. Cuán distantes estamos de los viejos maestros, ginecólogos y tocólogos, cuya ciencia parecía estar encerrada dentro de los estrechos límites de la pelvis femenina! En el más avanzado sentido del término la palabra especialización ha venido a significar no solamente el perfeccionamiento en un campo particular de los conocimientos médicos, sino la continua inquietud por todos los hechos notables que se establezcan en otros campos. Considerada así la Ginecología sobrepasa a todas las otras ramas: del concepto primitivo de las enfermedades de los órganos sexuales femeninos hemos llegado, franqueadas las etapas hormonal y nerviosa, al propio mundo interior; ya no será tan sólo en las alteraciones anatómicas, ni en los trastornos de funcionamiento en donde vamos a encontrar la causa de la enfermedad, sino que debemos penetrar más hondo en la personalidad de la paciente y darnos cuenta de sus conflictos sociales y económicos, de sus sentimientos e inquietudes, en una palabra de su vida afectiva. Desde este punto de vista la ginecología no es una ciencia de aparatos o sistemas, su alcance es mucho más vasto, en síntesis es la "ciencia de la mujer".

Una parte fundamental de este concepto, a menudo poco tenida en cuenta, es la constitución femenina. Brevemente definida la constitución es la suma total de las cualidades heredadas, alterada por las influencias ambientales; concierne no solamente a la conformación somática del individuo, especialmente sus órganos sexuales y los rasgos que ha heredado, sino también a su aspecto psíquico. Las peculiaridades psíquicas en la mujer cuentan tanto como sus órganos generativos especiales para la diferenciación biológica con su compañero de todos los tiempos. En nuestra patria, por factores ancestrales, de educación, de medio, de raza, cuando no de simple aislamiento del mundo que nos rodea, nuestras mujeres se han formado, y afortunadamente permanecen, como las reinas indiscutibles de los hogares; sobre esta base se han forjado sucesivas generaciones y sobre ella descansa inconmovible nuestra organización social y familiar. Pero la civilización no reconoce fronteras y ante su empuje cambian las costumbres y con ellas las organizaciones. No es para nosotros desconocida la influencia que su presencia en la oficina, en la fábrica, en el taller ha determinado para la mujer; estamos ahora ante un hecho nuevo: nuestras mujeres adquieren la plenitud de los derechos ciudadanos y se preparan a disputar al hombre las funciones del gobierno. No podemos predecir el impacto que estas conquistas determinen en nuestra organización social, pero sí podemos sospecharlas por lo que ha ocurrido en otros pueblos. De todas maneras, a fuer de médicos especializados en la ciencia de la mujer, no podemos permanecer indiferentes: estos aspectos novísimos del vivir femenino afectan su condición general e influyen en su función reproductiva. Deberíamos resolvernos a ayudar a la mujer a perseguir sus legítimos objetivos, pero velando siempre porque no se deformen sus características esenciales, porque cualquier cosa que afecte su felicidad y bienestar afecta la felicidad y el bienestar de toda la humanidad. 
Esta reunión, aparte de su inmenso valor intrínseco, se ve particularmente aprestigiada por la presencia de dos insignes representantes de la ciencia del viejo mundo; la Francia inmortal y la Italia eterna han querido estar presentes en este certamen: Señores Doctores Martial Dumont y Piero Malcovati, en nombre de la Sociedad Colombiana de Obstetricia y Ginecología os presento un efusivo saludo y una cordial bienvenida. Vuestra presencia en estas justas de la inteligencia nos confortan y es prenda segura que nos permiten anticipar el éxito de esta Convención.

Nos hemos dado cita en esta milagrosa ciudad de Barranquilla, la puerta de oro de Colombia, en donde, como lo dice su himno, "las sirenas de fábrica y taller son rumor arterial de su existir"; aquí no solamente el progreso avasallador, sino las más nobles emulaciones del espíritu; la medicina no podía ser una excepción: las semillas regadas por Miguel Arango y Anastasio del Río, por Martín Camacho y Rafael E. Pérez, para no mencionar sino unos pocos, cayeron en terreno fértil; prueba de ello esta brillante Sociedad de Obstetricia y Ginecología del Atlántico a cuyo entusiasmo debemos el que éste Congreso se haya convertido en realidad.

Ha querido la gentileza de su Presidente, Dr. Echeverri, que este modesto servidor de Uds. declare instalada la Convención: el objetivo es noble y bello, la inquietud científica muy grande, el entusiasmo desbordante y el ambiente franco y acogedor; bajo tan singulares auspicios comencemos a laborar por el bien de Colombia".

\section{Entrega de Diplomas como "Miembros Honorarios" de la Sociedad Colombia- na de Obstetricia y Ginecología a los Profesores José del Carmen Acosta y Pedro Nel Cardona.}

Para clausurar el Congreso y en ceremonia solemne el Sr. Presidente de la Sociedad Colombiana de Obstetricia y Ginecología, Prof. Bernal, pronunció las siguientes palabras:

Distinguidos colegas:

"La Sociedad Colombiana de Obstetricia y Ginecología ha querido cerrar con broche de oro el grandioso certamen que nos ha mantenido congregados, durante cuatro días, en esta ciudad juvenil que nos ha abierto sus brazos para acogernos; el objetivo no puede ser más noble: se trata de hacer justicia a las virtudes, de exaltar los merecimientos y de premiar la labor, no por callada menos trascendental de dos de nuestros más venerados maestros, notables científicos, beneméritos patriotas e inmejorables ciudadanos.

Y en verdad que la decisión que ha tomado la Sociedad que tengo el honor de presidir marca un hito en su existencia; fundada hace algo más de tres lustros se ha cuidado siempre al conceder sus distinciones, pero este celo ha llegado al máximo cuando se trata de otorgar su más preciado galardón: el título de "Miembro Honorario" no ha sido ostentado hasta ahora por persona alguna, por lo menos en lo nacional. Esta situación no podría prevalecer cuando contamos en el terreno de nuestra especialidad con figuras eximias que harían honor a la más exigente institución científica; los nombres de José del Carmen Acosta y de Pedro Nel Cardona brillan con luz propia en el panorama de la ciencia médica colombiana y así, cuando la actual Directiva les pro- 
puso, fueron aclamados por la Corporación en pleno: bien sabíamos que al honrarlos a ellos nos honramos a nosotros mismos.

Vidas hasta cierto punto paralelas, el uno en la altiplanicie, el otro en la montaña, ambos escalan por riguroso ascenso la más alta cima en la carrera del profesorado. Maestros por naturaleza y por temperamento prodigan sus enseñanzas en donde quiera que haya un entendimiento atento a comprenderlos y una voluntad dispuesta a seguirlos. Las más altas distinciones y los mayores honores que pueda ofrecer nuestra profesión han llegado a ellos sin inmutarlos ni envanecerlos: su mejor satisfacción deriva del deber cumplido y su única mira se orienta por el bien de Colombia.

Exaltar la vida y la obra de José del Carmen Acosta constituye un lugar común: lo hemos visto sabio y sencillo en la cátedra; celoso guardián del patrimonio moral de sus colegas en la más alta directiva de la Federación; brillante como rector de nuestra máxima Academia científica y arrogante cuando, ante países extraños, llevaba la vocería de la ciencia médica colombiana. Pero más lo hemos admirado por su vida inmaculada, ejemplo permanente de las más puras virtudes; jamás pasión alguna lo ha desviado de su camino recto; abanderado de toda causa justa su nombre se pronuncia siempre con respeto y, cuando en días aciagos para la patria, la soldadesca irrumpió en el sagrado recinto en donde José del Carmen Acosta vela por la salud de los enfermos bastó su sola presencia para hacer retroceder los fusiles de los jenízaros.

En Pedro Nel Cardona se resumen las glorias, las virtudes y el empuje de una raza que es orgullo de Colombia. Apasionado desde sus años mozos por el estudio de las enfermedades de la mujer, comprendió que para ser un destacado especialista se necesita ante todo ser un buen médico. Así lo vemos adiestrarse por los campos fascinantes de la Fisiología y de la Química Biológica, para llegar a ser luego un maestro de la Patología; y en clínica trajina primero con las enfermedades quirúrgicas generales antes de consagrarse definitivamente a la especialidad que tanto lo atrae. Como Profesor Titular de Ginecología de la Universidad de Antioquia en su cátedra se reverdecen los viejos laureles que ciñeran las frentes de Montoya y Flórez y de Gil. J. Gil, Profesor dinámico por excelencia, estudia, lee, investiga; escucha con atención a todo aquel de quien él cree que puede aprender algo; transmite su inquietud al grupo brillante de sus colaboradores y, en una escala nacional, lo hemos visto al través de los años como el principal animador de nuestras Convenciones.

Razón tiene pues la Sociedad Colombiana de Obstetricia y Ginecología al querer honrarse así misma colocando, como primeros, en el Olimpo de sus más altos valores, a estos dos varones insignes.*

Señor Profesor José del Carmen Acosta: desde la cumbre de vuestra gloriosa carrera contemplad el enjambre de vuestros discípulos que os rinden emocionado homenaje permitid al más modesto de todos ellos que, al entregaros este diploma, os exprese la admiración y el agradecimiento que todos os profesamos.

* La Revista Colombiana de Obstetricia y Ginecología hace suyas las palabras del Presidente de la Sociedad y se permite sumarse a quienes ven con orgullo la exaltación de los Profesores Acosta y Cardona. 
Señor Profesor Pedro Nel Cardona: aunque inicialmente concentrada a un Departamento privilegiado de la patria, vuestra labor se ha difundido por toda ella: este diploma que tengo el honor de entregaros es el reconocimiento a vuestras virtudes eximias que os destacan con brillo propio en el panorama científico de Colombia".

\section{Entrega de Diplomas como "Miembros Correspondientes" de la Sociedad Co- lombiana de Obstetricia y Ginecología a los Profesores Martial Dumont, de Lyon y Piero Malcovati, de Milán.}

A continuación el Prof. H. E. Bernal pronunció las siguientes palabras:

"La Sociedad Colombiana de Obstetricia y Ginecología ha querido también enriquecer su patrimonio incorporando entre sus miembros a los dos insignes representantes de la ciencia francesa e italiana que han querido honrarnos con su presencia en esta Convención. A cualquiera de ellos les sobran títulos y merecimientos para pertenecer a nuestra Sociedad y no pretendo fatigar a los oyentes con la enumeración de todos ellos; básteme con recordar que, tras de una cimentada trayectoria científica, el Dr. Dumont ha llegado al ambicionado cargo de "Profesor Agregado de Obstetricia en la Facultad de Medicina de Lyon" y el Profesor Malcovati es "Director del Instituto Provincial de la Maternidad de Milán”.

Lamento no contar con la presencia del profesor Dumont, cuyo diploma le será remitido oportunamente.

Señor Dr. Piero Malcovati:

Constituye para mí un gran honor el hacerle entrega de este Diploma: con él se lleva Ud. la admiración y el afecto de sus colegas de Colombia.

No podría terminar estas palabras sin expresar una vez más, a nombre de todos los colegas reunidos en esta Convención, la grata impresión que nos levamos de esta ciudad adolescente, alegre y acogedora, y el profundo reconocimiento para los organizadores del grandioso certamen. Señores Miembros de la Directiva de la Sociedad de Obstetricia y Ginecología del Atlántico: en la imposibilidad de encontrar otro vocablo que exprese mejor los sentimientos que nos animan nos limitamos a decirles gracias".

\section{ASISTENTES AL CONGRESO Y PATROCINADORES:}

Asistieron al Congreso los siguientes Doctores procedentes de varias ciudades del país.

BOGOTA - Doctores: Martín Méndez, Hernando Amaya León, Eduardo Arévalo Burgos, Miguel A. Fernández-Bastidas, Guillermo López-Escobar, Gustavo Riaño, Jorge Amorocho Carreño, Alvaro Espinosa, Jesús A. Gómez Palacino, Héctor Enrique Bernal, Fernando Sánchez Torres, Héctor Rozo Pardo, Rodulfo Camero C., Hernando Osorio, Carlos Martínez S., Roberto Ver- 
gara T., Hernando Navas A., Luis Salazar D., Manuel Aristizábal, Alvaro Fonnegra Miramón, Florentino de la Cruz, Alejandro Molina Vásquez, Alfredo Izquierdo, Humberto Correa, Rafael González, Carlos Arturo Goubert, Alberto Becerra y Becerra, Francisco Pardo Vargas, Roberto C. Casas, Hernando Caicedo, César Mendoza Posada, Jesús M. Forero Neira, Manuel J. Gómez Palacino, Carlos Roberto Silva Mojica, Miguel Orticochea.

BARRANQUILLA - Doctores: José A. Rosillo, Francisco Sales Sales, Eduardo Ydrovo, Hernando Echeverry, José Garrido Gómez, Fabián Jiménez Arango, Hugo Flórez Moreno, Eduardo Acosta Bendeck, Fernando Mercado F., Orlando Puccini B., Paolo Giamuzzi, Eugenio Cambiari, Liuba de Sohmulson, Eloy Barrios Herrera, Rafael Alvear C., Orlando Gutiérrez L., Fernando Navas U., Abei Karim el Ch., H. Cuentas Cepeda, Raúl Villalobos Rojas, Gonzalo Martínez Ariza, Luis Chicre, Adalberto Amador, David Hleap Felder, Ricardo Gaviria Torres, Héctor Fernández Angulo, Carlos Hernández S., Manuel Santana Saicedo, Luis E. Toscano, Gonzalo Cabrera Forero, Abdón Fadul P., Manuel Barrios S., Leonello Marthe Z., Jorge Tello Quijano, José de J. Navarra, Enmanuel Martes, Rafael Algarin M., Cristóbal González Navarra, Sebastián M. Alcalá, Ezequiel Polo P., Adolfo Cuentas Cepeda, Félix Barraza Pallares.

BARRANCABERMEJA - Doctores: Leonidas Oyaga Patiño, Dagoberto Rocha, Jaime Vieira Jaramillo, Luis A. López B., Alfredo Esper.

BUCARAMANGA - Doctores: Isaías Arenas Buenahora, Manuel G. Rangel, Enrique Sánchez Puyana, Fabio Durán Velasco.

CALI - Doctores: Jorge Escobar Soto, Saulo Muñoz, Geraldo Chadid Samur, Humberto Caicedo Perea, Julio Orozco, Reinaldo Mora, Javier Cabrera, Alvaro Cuadros.

CARTAGENA - Doctores: Napoleón Franco Pareja, Andrés J. Tarra, Antonio María Martínez.

CIENAGA DE ORO - Doctor Rufo Mendoza.

CODAZZI - Doctor Miguel A. de la Hoz Pallares.

CUCUTA - Doctores: Alberto Duarte Contreras, Alfredo Landines Salamanca, Fidoly Rangel Rozo, Sergio Lamus Guerrero, Pablo Emilio Ramírez Calderón, Reinaldo Omaña, Humberto Failacé.

EL BANCO - Doctores: José A. Cartissoz Gil, Néstor Casado Ravelo, Nazario Fraija Larios.

FUNDACION - Doctor Guzmán Granados de la Hoz.

IBAGUE - Doctores: Jaime Rengifo Pardo, Rafael Carvajal Gómez, Hernán Arbeláez.

MANIZALES - Doctor Oscar Acevedo F.

MEDELLIN - Doctores: Fernando Cardona Arango, Pedro Nel Cardona, Alfonso Jubis H., Gustavo Isaza Mejía, Gonzalo Vásquez, René Díaz Correa, Benecio Gaviria, José Mario Posada, Eduardo Yepes Pineda, Antonio Beltrán Galindo, Alberto Betancourt, Leonardo Alvarez, Jorge Restrepo, Ramiro Zúñiga, Antonio Vélez, Luis Tirado Vélez, Jaime Botero Uribe. 
MONTERIA - Doctores: Ricardo Bechara, Octavio Morillo, Mario Giraldo, José María Cabrales.

NEIVA - Doctores: G. A. Durán Cabrera, Rafael Núñez, Carlos López López, Fabio Sanz Hurtado, Hugo García Vélez, Abel Villegas.

PAMPLONA - Doctor Enrique Hernández Pérez.

SANTA MARTA - Doctores: Ismael Noguera Gómez, Julio Martínez A., Luis J. Gámez del Valle.

SINCELEJO - Doctor Francisco Jaraya.

VALLEDUPAR - Doctores: Hermes Araméndiz Oñate, Esteban Cuello, Cristóbal J. Celedón Ramírez.

PLATO - Doctor Leonardo Bustamante.

SAN JUAN DEL CESAR - Doctores: Enrique C. Gutiérrez, Juan H. Marengo.

MAGANGUE - Doctor Wadi Cure.

ZIPAQUIRA - Doctor Guillermo Huertas González.

SUCRE - Doctor Diógenes Jiménez.

SAN MARCOS - Doctor Isaías Carriazo Ealo.

ZAMBRANO - Doctor Lizardo Ochoa.

A la feliz realización del Congreso contribuyeron las siguientes firmas:

Laboratorios Fixalia, Laboratorios Life, Merck Sharp \& Dohme, Laboratorios Quibi, Laboratorios Lilly, Laboratorios Abbot, Parke Davis Interamerican Corp., Distribuidora Frosst, Shering Corporation (Undra Ltda.), Laboratorios Ayers, Química Premier, Instituto Bio-Químico, Laboratorios Upjohn, Laboratorios Pfizer, Laboratorios Bristol (Stand y Té para las Sras.), Laboratorios Labfarmi, (filmación del Congreso), Químicas Knoll de Colombia, Laboratorios Specia (invitados extranjeros), Productos Roche, Arlington Funk Laboratories de Colombia, Laboratorios Cup, Laboratorios Hormona, Laboratorios Winthrop, Laboratorios Incobra, (Cocktail de clausura), Laboratorios Wyeth (Gallardetes, afiches, insignias etc.), Productos Erba (Lafrancol Ventura Pauly) Programas e invitados extranjeros; Laboratorios Squibb (Carteras), Cicolac (Stand de gustación, y aviso en El Tiempo alusivo al Congreso), Schering Alemana (Cocktail).

\section{TERCER CONGRESO MUNDIAL DE FERTILIDAD Y ESTERILIDAD}

Amsterdam - Junio de 1959

\section{Informe enviado por el Dr. Aristóbulo Cruz A. del Centro Médico del Quindío - Armenia}

En este congreso se presentaron numerosísimos y muy interesantes trabajos sobre los siguientes temas: Problemas psicosexuales en esterilidad; Muerte 
embrionaria; Morfología, fisiología y bioquímica del esperma; Factores hormonales y vitaminas en fertilidad y esterilidad; Valor relativo de las técnicas para el estudio de las funciones endocrinas en esterilidad; Esterilidad femenina y masculina en general; Investigaciones básicas y reproducción animal.

A continuación se transcriben los resúmenes de los trabajos más interesantes.

FACTORES PERSONALES EN ESTERILIDAD. Por H. Blijham, de Groningen, Holanda.

El autor dice, que en un estudio psicosomático e integral de los problemas en los matrimonios estériles, conduce a considerar al paciente como una entidad, y como una unidad indivisible, con aspectos somático, psíquico y social. Cita gran número de trabajos que muestran casos de esterilidad como una manifestación psicosomática. Hace mención de concepciones después de una adopción, después de un tratamiento psicoanalítico, después de un cambio de vida, después de unas vacaciones, de una recreación, después de un tratamiento para frigidez, después de una intervención quirúrgica cualquiera, después de un examen ginecológico, después de conseguir un empleo. Hace comparaciones con la pseudopreñez, con los disturbios menstruales psicogénicos y otros síndromes ginecológicos. Observaciones paradójicas, por ejemplo, preñez a pesar del gran temor de él, dice que se pueden interpretar como contraste entre el deseo consciente y subconsciente. En la literatura se mencionan también varios mecanismos psicofísicos: Factores neuro-endocrinos. Factores musculares en las trompas y el útero, Factores cervicales, Factores vasculares y Factores bioquímicos-bioeléctricos.

En relación con los Factores Neuroendocrinos, se debe tener presente el eje corteza-diencéfalo-hipotálamo-hipófisis anterior-epífisis y ovario. A este grupo pertenece la ovulación prematura, en la que el huevo no es suficientemente viable o es insuficientemente espermatotrópico, y los casos de ciclos anovulatorios. Se atribuyen a Factores Musculares los espasmos tubáricos, la diskinesia tubárica, la motilidad uterina irregular y la hiperestimulabilidad uterina. Se citan varios ejemplos de espasmos tubarios interpretados erróneamente como oclusiones tubáricas y esto puede sugerir una posible causa de embarazo ectópicos en mujeres estériles. Entre los Factores Cervicales, el principal es la excesiva excreción cervical (alcalina) que favorece las infecciones vaginales. Los Factores Vasculares, tienen relación con los problemas de congestión pélvica, la que puede alterar la función ovárica, especialmente la maduración folicular; como resultado de esto pueden venir disturbios secundarios, se puede desarrollar fibrosis ovárica. Los factores emocionales pueden tener también influencia en la fertilidad y esterilidad a través de estos mecanismos psicofísicos. El autor dice que un estudio de los aspectos psicopatológicos y psicodinámicos, revelan varias ansiedades relacionadas, por ejemplo, preñez y sexualidad. Hay también sentimientos de culpa. La relación psicosexual a menudo no es satisfactoria. La agresión puede ser un factor. En algunos casos la esterilidad funcional tiene que ser interpretada como una defensa contra el stress que trae consigo la preñez y la maternidad para algunas mujeres. El autor anota que es poco lo que se ha escrito en cuanto a factores psicogénicos en el hombre. Michael publicó un estudio muy completo a este respecto y concluye 
que para la fertilidad son de mucha importancia unas relaciones sexuales adecuadas, armoniosas y sinérgicas y opina que los disturbios psicogénicos sexuales son causa frecuente de subfertilidad o infertilidad en los hombres.

Varios autores han intentado describir las características del paciente estéril. Se han aplicado diferentes principios terapéuticos: sugestivo, relajante, analítico; en tales tratamientos tienen capital importancia las relaciones médicopaciente. La profilaxis es posible y necesaria. Se llama la atención de los peligros del tratamiento del paciente funcionalmente estéril, cuya labilidad psíquica puede hacer peligrosa la preñez y la maternidad. El autor anota que injustificadamente se ha puesto mayor atención a la esterilidad femenina que a la masculina, en relación con los factores psicogénicos y que no hay presumiblemente un límite exacto entre el "grupo orgánico" y el "grupo funcional".

\section{EL DIAGNOSTICO HORMONAL DE LA DISFUNCION PLACENTA- RiA CONDUCE AL DE LA MUERTE FETAL. Por el Prof. B. Zondek. Jerusalén, Israei.}

El Autor sostiene que la producción de hormonas sexuales por la placenta, demostrada por su titulación urinaria, se puede usar como "Test" sobre función y disfunción placentaria (rcversible o irreversible). La titulación de la gonadotropina coriónica, recomendada como prueba de la preñez reciente, ha probado tener su importancia en la terapia para la amenaza de aborto. La titulación del estriol urinario, se recomienda como prueba de la función placentaria en la preñez avanzada (a partir de la 20 a semana). Los estrógenos excretados por la orina contienen $90 \%$ de estriol, y solamente $10 \%$ de estrona y estradiol. La determinación del estriol, es especialmente adecuada como investigación funcional.

La determinación cuantitativa del estriol se obtiene por el método fotofluorométrico. Las fluctuaciones en la eliminación urinaria del estriol indican disfunción placentaria; la concentración puede bajar hasta un $70 \%$. Si vuelve a subir, quiere decir que se trata de una disfunción placentaria reversible, pero si la concentración continúa descendiendo (permaneciendo por debajo de mil unidades por 24 horas) quiere decir que hay una disfunción placentaria irreversible que conduce a la muerte del feto. En casos de toxicosis gravídica las fluctuaciones son de mayor importancia pronóstica que en el embarazo normal. El "test'. hormonal informa acerca de la disfunción placentaria en relación con la muerte fetal y el aborto.

DIAGNOSTICO DE LA MUERTE INTRAUTERINA EN EL ULTIMO TRIMESTRE DE EMBARAZO. Por B. S. Ten Berge, de Groningen, Holanda.

$\mathrm{El}$ autor dice que el diagnóstico de la muerte fetal intrauterina puede ser difícil. Hay duda cuando no hay movimientos a pesar de oír ruidos cardíacos fetales. El signo de que el tamaño de la matriz no corresponde al tiempo de embarazo tiene poco valor en la toxemia, en las lesiones cardíacas maternas o en las anormalidades fetales congénitas. El signo de Spalding es positivo solamente después de algún tiempo. Sostiene el autor, que la excreción de estriol cae súbitamente después de la muerte fetal y que los valores absolutos son de menor importancia que la caída súbita del estriol en controles seriados. La razón de esto es la supresión de la acción capilar en las vellosidades, por lo 
cual el transporte de sangre en los espacios entre las vellosidades se disminuye. El Autor practicó la localización de la placenta en 11 embarazadas. Para esto inyecta 5 u. c. de seroalbúmina humana yodada y radioactiva por vía intravenosa. Luego se mide con un contador la radioactividad sobre el abdomen y el corazón. Previamente se da una solución de yoduro de potasio para proteger el tiroides de la madre y el feto. La radioactividad medida en el tiroides del niño fue ausente o casi nula. La radioactividad abdominal, sobre la región placentaria, se encontró casi tan alta como sobre el corazón de la madre. Cuando el feto está muerto no es posible la localización de la radioactividad placentaria, debido a la suspensión de la actividad capilar de las vellosidades.

\section{ABORTO HABITUAL DEBIDO A INCOMPETENCIA DEL ISTMO UTE- RINO. Por R. Palmer y E. Palmer. París.}

Dicen los Autores, que la incompetencia del istmo uterino es una de las causas más frecuentes de aborto entre el $4^{\circ}$ y el $6^{\circ}$ mes. La etiología es traumática en la mayoría de los casos, aunque no siempre. El diagnóstico se puede sospechar por las características del aborto (prolapso y ruptura primaria de las membranas, feto vivo). Algún tiempo después del aborto se puede confirmar el diagnóstico, por la introducción, sin resistencia, de una bujía de Hegar No 8 y por histerografía. La traquelorrafía extensa (Palmer y Lacome, 1948) es aplicable en casos donde la laceración se ha extendido hasta el istmo (éxito en 4 de 5 casos). La istmorrafia (Lash 1950) ha dado resultados inconstantes (éxito en 9 de 16 casos). La operación de Shirodkar (1951) que usa una banda de fascia lata es dispendiosa y hace necesaria la cesárea. Desde 1953, el Autor ha usado una operación de Shirodkar modificada (Palmer, 1957) empleando crin de Florencia alrededor de la porción supravaginal del cuello, preferiblemente en el tercer mes de embarazo. En la iniciación del parto se secciona la crin y el alumbramiento se verifica normalmente.

EL TIEMPO DE LA OVULACION Y SU VALOR PARA LA CORRECTA DETERMINACION DE LA FECHA DEL NACIMIENTO Y LA DURACION DEL EMBARAZO. Por H. H. Knaus, de Viena.

Anota el Autor, que en todos los países civilizados del mundo, el tiempo del nacimiento se determina según el método recomendado hace 125 años por el obstetra alemán Nagele en Heidelberg. Esta determinación se basó partiendo de la fecha del primer día de la última menstruación a la cual se quitan 3 meses y se agregan 7 días. El Autor dice que no es correcto este método, pues la concepción nunca puede ocurrir el primer día de la menstruación, si no mucho después. Dice que la cuenta se debe hacer quitando 3 meses a la fecha de la ovulación individual; y como el tiempo de la ovulación se puede extender en un período más o menos largo, la fecha del nacimiento no se puede predecir sobre un día, sino dentro de un período de varios días.

\section{ESTUDIOS SOBRE LA FERTILIZACION IN VITRO DE OVULOS HU-} MANOS Y DE MAMIFEROS. Por Hayashi, de Tokio.

Dice el Autor, que el propósito de este estudio, fue investigar la fertilización in vitro y analizar las mejotes condiciones para este procedimiento. 
Los óvulos se obtuvieron de mujeres, conejas, vacas, cochinillas y perras.

Se emplearon espermatozoides de las mismas especies.

Los óvulos se obtuvieron por laparotomía en el tiempo de la ovulación. Se emplearon los siguientes medios de cultivo:

Solución A

Solución B

Solución de Ringer

Suero sanguíneo

Glucosa

Fructuosa

Penicilina

(o cloramfenicol 300 mgr.)

$\begin{array}{rlr}90 \mathrm{ml} . & \text { Solución de Ringer } & 100 \mathrm{ml} . \\ 10 \mathrm{ml} . & \text { Glucosa } & 5 \mathrm{~g} . \\ 5 \mathrm{~g} . & \text { Fructuosa } & 5 \mathrm{~g} . \\ 5 \mathrm{~g} . & \text { Penicilina } & 100.000 \mathrm{u} .\end{array}$

100.000 u. Extracto tubárico.

Se observaron con microscopio de contraste de fase a $37^{\circ} \mathrm{C}$ de temperatura. Las divisiones (de 2 , de 4 , de 8 células) se vieron fácilmente en las conejas, las ratas y las vacas; pero fue más difícil en las mujeres, las cochinillas y las perras. Para obtener suficientes divisiones se cree que tenga mucha importancia la esterilización y el cambio de medio de cultivo.

\section{INFORMES}

\section{INFORME DE LA DIRECCION DE LA REVISTA A LA SOCIEDAD COLOMBIANA DE OBSTETRICIA Y GINECOLOGIA}

\section{Año 1959}

Al finalizar nuestro tercer año de ejercicio como Directores de la Revista Colombiana de Obstetricia y Ginecología, presentamos a la consideración de la Asamblea General de la Sociedad, reunida en pleno, el informe reglamentario sobre labores llevadas a cabo durante 1959; año para el cual fuimos elegidos por la Junta Directiva de la Sociedad y con la cual nos obliga una deuda de gratitud en la persona de su Presidente Profesor Dr. Héctor Enrique Bernal por la confianza ilimitada depositada en nosotros durante el ejercicio de su mandato.

Como afortunadamente, todos los suscriptores y socios han tenido la oportunidad de juzgar por sí mismos, uno por uno todos los seis números que formaron el Volumen Décimo de nuestra Revista; simplemente trataremos de destacar los principales hechos científicos y económicos que a través de su salida han acaecido.

Siguiendo el derrotero trazado con anterioridad, todos los artículos publicados (excepción desde luego de los resúmenes) fueron de carácter inédito y de reconocido valor científico, siendo en su mayoría aportes hechos a Congresos nacionales, o contribuciones en las ininterrumpidas e interesantes sesiones científicas de la Sociedad. Igualmente, la creación de secciones especiales tales como las denominadas "Sección de Endocrinología Obstétrica y Ginecológica" y "Sección de Obstetricia Sicosomática"; tuvo como finalidad, dar forma de 
expresión a una creciente literatura científica sobre el tema o a interesantes cursos para post grado, llevados a cabo con destacadas figuras científicas foráneas.

De la importancia de tales "simposios" no hemos dudado en ningún momento, por estar convencidos cada vez más, del papel didáctico preponderante que la Revista debe ejercer en lugares apartados de los centros de estudio, en donde el médico está la mayoría de veces incapacitado para orientar el ejercicio de la especialidad; aclarándole conceptos o ayudándole a adquirir nuevas ideas que le harán más científica su labor profesional.

En esta forma, queremos resaltar, que no solo la Revista ha tenido durante los últimos años como finalidad, la publicación de la obra científica nacional, sino la orientación práctica de aquellos colegas que ya le reconocen una utilidad, y que en especial fuera de Bogotá reclaman su envío de manera insistente.

Como es natural, la mayoría de las contribuciones fueron enviadas por uno de los tres centros universitarios nacionales de mayor estímulo investigativo: Bogotá, Medellín o Cali; pero ya se ha podido observar en el pasado volumen la aparición de nuevos escritores nacionales y de autores extranjeros, que han tenido a bien honrarnos con su colaboración.

La campaña sostenida y planteada en no pocas oportunidades ante la Sociedad por esta Directiva, en relación con el estímulo a la investigación en centros hospitalarios de otras capitales, pese a las fallas técnicas que inicialmente pudieran tener los trabajos elaborados, no dudamos en aconsejar sea continuada por la directiva que nos suceda; pues por alguna razón, la Revista como la Sociedad al denominarsen colombianas aclaran su carácter inminentemente nacional.

Con motivo del IV Congreso Colombiano de la especialidad celebrado en la ciudad de Barranquilla hace apenas unos cuantos días, la Dirección de la Revista siguiendo la costumbre establecida programó una nueva Asamblea Nacional con todos los miembros de su comité de redacción. En la agenda elaborada para tan importante reunión, figuró un punto relativo a la elaboración de reglamento de Revista el cual una vez puesto en consideración de la Sociedad deberá servir de base para una mayor autonomía de nuestra publicación. Con tal objeto y en aquella ocasión fué aprobada una proposición en la cual además de dar pleno apoyo a las directivas en ejercicio, autorizó a la Dirección para elaborar el reglamento en cuestión.

Hoy día el reglamento está elaborándose, y corresponderá a las próximas directivas de la Revista presentar a la junta para su estudio un proyecto de reforma estatutaria que les permita regirla por sus propias normas. En esta forma la Revista Colombiana de Obstetricia y Ginecología, sin dejar de ser el órgano publicitario de la Sociedad, tendrá una mayor iniciativa y suficiente movilidad, que no dispone en la actualidad por falta de una reglamentación apropiada, acorde con su ya notorio desarrollo.

En cuanto a la parte financiera, debemos informar a la Sociedad, que dado el incontrolable aumento del costo de vida reflejado en el alza de precios de publicación y aunque superada la etapa de reajuste planteada en nuestro informe del año 1958; el Vol. X con un aumento de páginas sensiblemente igual 
al volumen anterior tuvo un costo mayor que aquel; habiéndose notado que el aumento de los ingresos compensaron tal alza, hasta el punto de poder presentar ante Uds. un superavit.

En esta foima los ingresos de acuerdo con el balance presentado a la junta, correspondicron aproximadamente en un $70 \%$ más o menos a propaganda comercial y un $30 \%$ restante a suscripciones y reimpresos.

Cabe resaltar al llegar a este punto, el hecho que la Revista Colombiana de Obstetricia y Ginecología, es hoy la única revista médica colombiana que tiene suscripciones pagas, las cuales si bien como Uds. lo acaban de oír, no cubren el valor de los gastos de edición, sí han ido en aumento a partir del año 1957 en que se iniciaron. Sinembargo, hacemos de nuevo un llamamiento a todos los miembros de $\mathrm{l}_{\boldsymbol{A}}$ Sociedad para que directa o indirectamente contribuyan a aumentar el número de suscripciones logrando por este medio estimular su mejoramiento.

Para terminar el informe económico debemos aclarar que nuestra última gestión en este campo ha sido la financiación de los dos próximos números del Vol. XI, en los que aparecerán trabajos presentados al IV Congreso reunido en Barranquilla. En efecto, hemos obtenido, a más del $20 \%$ del valor de las inscripciones a dicho Congreso, un contrato de edición con la casa editorial Omnia, la cual, amablemente y como contribución voluntaria facilitó la operación.

La organización de la hemeroteca (o biblioteca de revistas) de la Sociedad continúa siendo un problema a nuestro modo de ver, desgraciadamente ya de larga data. Hace algunos años la Directiva de la Revista teniendo en cuenta la gran utilidad que podría prestar a todo el que fuera a consultar el gran número de revistas que llegan como canje, aconsejó y obtuvo de la junta de aquel entonces el nombramiento de bibliotecario, el cual a nuestro modo de ver debería hacer parte de la junta directiva y por lo tanto elegible al nombramiento de ésta.

El distinguido profesional que ocupó por primera vez esta posición organizó los ficheros y obtuvo un inventario total de existencias; pero después de cumplirse su ejercicio tal organización cayó en completo abandono.

No siendo despreciable tan abundante material de consulta debidamente clasificado, aconsejamos que la próxima junta teniendo en cuenta las razones aducidas, ponga los medios para reorganizar la biblioteca que aunque en su mayoría formada por revistas, es parte del patrimonio de la Sociedad y tiene por lo tanto la obligación de preservar.

Para terminar proponemos, para un futuro y para que sea considerado por la Sociedad a través de su junta directiva y desde luego por la próxima Dirección de la Revista, dos puntos que nos parecen de importancia. El primero el proyecto de dotar a la Revista de una carátula de material de mejor calidad, hecha a base de cartulina satinada importada (hoy día no se consigue en el comercio) del mismo color amarillo carácterístico y cuyo costo podría ser sufragado por un préstamo de la misma Sociedad.

Segundo: la necesidad ya urgente, de dotar a la Sociedad Colombiana de Obstetricia y Ginecología de un emblema distintivo, que para la Revista y es- 
pecialmente para el material impreso de propaganda con motivo del próximo Congreso Latino-Americano, tendría señalada importancia; y que perfectamente se podría lograr, considerando ideas y proyectos de los mismos socios, estableciendo si es el caso un premio al mejor logrado, previo dictamen de una junta nombrada para calificarlo.

En esta forma damos por terminada una vez más la labor en la Dirección, declarando nuestro reconocimiento para con las juntas que tuvieron a bien reelegirnos y en especial hacia el Sr. Presidente, Profesor Héctor Enrique Bernal con quien tratamos de colaborar ampliamente durante el período que hoy toca a su fin.

\section{Dr. Miguel A. Fernández-Bastidas - Dr. Guillermo López-Escobar}

\section{para ecuanimidad...}

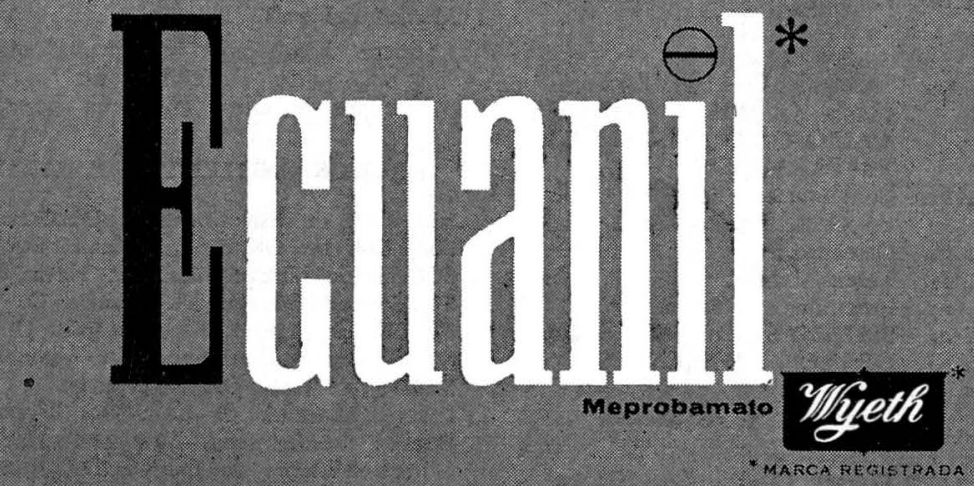

Tranquiliza la mente y restablece el tono muscular.

Permite un sueño normal, reparador, exento de pesadillas.

No tiene contraindicaciones. 